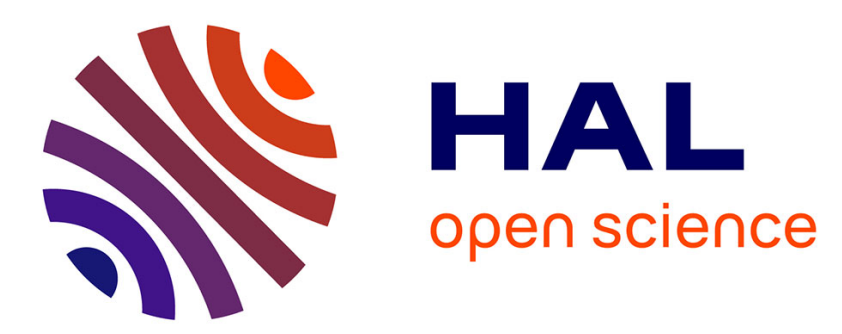

\title{
A Robust Hit-or-Miss Transform for Template Matching in Very Noisy Astronomical Images
}

Benjamin Perret, Sébastien Lefèvre, Christophe Collet

\section{To cite this version:}

Benjamin Perret, Sébastien Lefèvre, Christophe Collet. A Robust Hit-or-Miss Transform for Template Matching in Very Noisy Astronomical Images. Pattern Recognition, 2009, 42 (11), pp.2470-2480. 10.1016/j.patcog.2009.02.013 . hal-00512735

\section{HAL Id: hal-00512735 \\ https://hal.science/hal-00512735}

Submitted on 31 Aug 2010

HAL is a multi-disciplinary open access archive for the deposit and dissemination of scientific research documents, whether they are published or not. The documents may come from teaching and research institutions in France or abroad, or from public or private research centers.
L'archive ouverte pluridisciplinaire HAL, est destinée au dépôt et à la diffusion de documents scientifiques de niveau recherche, publiés ou non, émanant des établissements d'enseignement et de recherche français ou étrangers, des laboratoires publics ou privés. 


\title{
A Robust Hit-or-Miss Transform For Template Matching Applied to Very Noisy Astronomical Images.
}

\author{
B. Perret \\ S. Lefèvre \\ Ch. Collet \\ Laboratoire des Sciences de l'Image, de l'Informatique et de la Télédétection (LSIIT), \\ Université de Strasbourg, France UMR CNRS 7005
}

\begin{abstract}
The morphological Hit-or-Miss Transform (HMT) is a powerful tool for digital image analysis. Its recent extensions to grey level images have proven its ability to solve various template matching problems. In this paper we explore the capacity of various existing approaches to work in very noisy environments and discuss the generic methods used to improve their robustness to noise. We also propose a new formulation for a fuzzy morphological HMT which has been especially designed to deal with very noisy images. Our approach is validated through a pattern matching problem in astronomical images that consists of detecting very faint objects: low surface brightness galaxies. Despite their influence on the galactic evolution model, these objects remain mostly misunderstood by the astronomers. Due to their low signal to noise ratio, there is no automatic and reliable detection method yet. In this paper we introduce such a method based on the proposed hit-or-miss operator. The complete process is described starting from the building of a set of patterns until the reconstruction of a suitable map of detected objects. Implementation, running cost and optimisations are discussed. Outcomes have been examined by astronomers and compared to previous works. We have observed promising results in this difficult context for which Mathemat-
\end{abstract}

Email addresses: perret@lsiit.u-strasbg.fr (B. Perret), lefevre@lsiit.u-strasbg.fr (S. Lefèvre), collet@lsiit.u-strasbg.fr (Ch. Collet) 
ical Morphology provides an original solution.

Key words:

Hit-or-Miss Transform, Template Matching, Mathematical Morphology, Noisy environment, Astronomy, Low Surface Brightness Galaxies

\section{Introduction}

Template matching is a subject of interest in many computer vision processes and more generally in digital image analysis. In a general formulation it consists of searching for occurrences of a predefined object in an image. The objects of interest can have several different visual appearances which lead to several patterns sought in the images.

Among the methodologies to be used for pattern matching in digital images, mathematical morphology is particularly relevant since it performs spatial based image analysis relying on a pattern called structuring element (SE). The HMT is a well known morphological operator dedicated to template matching. Initially defined for binary images, it has been extended recently to greyscale images [1].

The use of the morphological HMT to perform template matching has grown during recent years. Different formulations have been proposed and their application to various domains (medical imaging [2] [3], remote sensing [4][5], character recognition [6], face localisation [7]) have proven their ability to provide a generic pattern matching method. However it lacks of robustness to noise and thus cannot be yet considered as a valid option for object detection in noisy astronomical images, even if some promising results have already been obtained in other challenging tasks such as galaxy segmentation and classification [8].

In this paper we propose a new formulation of the morphological HMT for grey level images. This formulation leads to a fuzzy result and can be applied even on very noisy images with a signal to noise ratio below $0 d B$. An application to detect faint objects: low surface brightness (LSB) galaxies [9] in astronomical images is also proposed. Detecting these objects is a very topical problem since astronomers lack of information about them. It also well fits our interest of adapting the HMT to deal with very noisy environment for several reasons. The shape and the luminance profile of LSB galaxies are known, thus we can easily define a set of templates representing the various visual appearances they can take. However, their detection is a hard task as 
astronomical images have very heterogeneous content. The pixel dynamic can be very large with a brightness ratio between faintest and brightest objects coming up to 1:1000. The size variability of objects present in astronomical images can also be huge with objects covering only a few pixels to the whole image. Moreover, due to projective effects, different objects may overlap. Finally, to observe such faint objects as LSB galaxies, astronomers have to use long exposition time to collect as many photons as possible, thus leading to also collect a lot of noise.

This paper is organized as follows. First we briefly recall the basics of grey level morphology. Then we discuss the existing approaches for grey level HMT and their relative robustness to noise. We introduce a new formulation of the fuzzy HMT and give a methodology to involve this formulation in template matching applied to the detection of LSB galaxies in astronomical images. Finally results of this application are discussed and a comparison with existing astronomical results is proposed.

\section{Review of Existing Hit-or-Miss Transforms}

We first recall the basics of grey level morphology, using the notations given by Naegel et al [10]. Let $E$ be a digital space (e.g. $\left.E=\mathbb{Z}^{n}\right)$. Let $T$ be a set of grey levels. We require $T$ to be a complete lattice with respect to the order " $\leq$ " [11][12]. We will next assume that $T=\mathbb{R} \cup\{+\infty,-\infty\}$ or $T=\mathbb{Z} \cup\{+\infty,-\infty\}$. We denote $\perp$ and $T$, respectively, the lowest and greatest elements of $T$.

Let $I$ and $G$ be two functions of $T^{E}$, the set of functions going from a subspace of $E$ to $T$. We call the support of $I(\operatorname{supp}(I))$ and dual support $\left(\operatorname{supp}^{*}(I)\right)$ the set of points where $I$ is strictly above $\perp$ and respectively under $\top$. Dilation $\oplus$ and erosion $\ominus$ of $I$ by $G$ at every point $p \in E$ are then given by:

$$
\begin{aligned}
& (I \oplus G)(p)=\sup _{k \in \operatorname{supp}(G)}(I(p-k)+G(k)) \\
& (I \ominus G)(p)=\inf _{k \in \operatorname{supp}(G)}(I(p+k)-G(k))
\end{aligned}
$$

You can notice that these formulas can lead to disinclination like $+\infty$ plus $-\infty$. To keep consistency $+\infty$ plus $-\infty$ must be valued as $-\infty$ in Eq. 1 and as $+\infty$ in Eq. 2. Further readings about grey level morphology can be found in [13][14]. We also define the dual $G^{*}$ of the function $G \in T^{E}$ by:

$$
G^{*}: E \longmapsto T
$$




$$
p \longrightarrow-G(-p)
$$

We now recall the principles of the HMT. It consists of searching for all pixels in an image which have a desired neighbourhood. It requires two SEs: the first one called foreground SE describes the shape and the second one called background SE is used to describe the spatial neighbourhood of this shape. In the binary case the interpretation of background and foreground is straightforward: the foreground is a set of pixels which has to be present in the image and the background is a set of pixels which has to be present in the complement of the image (see Fig. 1 for example).
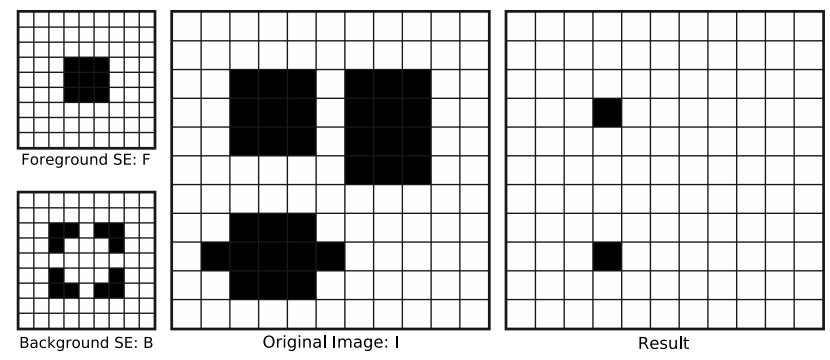

Figure 1: Example of application of binary HMT to detect $3 \times 3$ squares with possible extension along edges. The top left image represents the foreground SE: $F$, origin of the image is at the centre of the square. The bottom left image is the background SE $B$, with same origin as $F$. The middle image is the original image $I$. And the right image is the result of the HMT applied on $I$ with SEs $F$ and $B$.

The extension of HMT to complete lattice requires to define foreground and background in such a general case. Thus several approaches have been proposed [15][10][3][16]. We will now present these approaches and discuss their resistance to noise in images with very low signal to noise ratio $(S N R \leq$ $0 d b)$, the SNR being defined as signal energy divided by noise energy and is expressed in logarithmic scale:

$$
S N R=10 \log _{10}\left(\frac{\sigma_{s}^{2}}{\sigma_{n}^{2}}\right) d b
$$

with $\sigma_{s}$ and $\sigma_{n}$ being respectively the standard deviation of signal and noise.

\subsection{Existing formulations of grey level HMT}

We now introduce the various existing approaches to grey level HMT. For each operator, we discuss its ability to deal with poor SNR which is of primary importance for template matching in noisy images. 
Let $I, F, B \in T^{E}, F$ and $B$ are the foreground and background SEs such that $F \leq B$ (i.e. $\forall p \in E, F(p) \leq B(p))$, I stands for the image.

\subsubsection{Ronse's and Soille's HMT}

The definition of Ronse's HMT given in [10] is:

$$
\operatorname{RHMT}_{[F, B]}(I)(p)=\left\{\begin{array}{cl}
(I \ominus F)(p) & \text { if }(I \ominus F)(p) \geq\left(I \oplus B^{*}\right)(p) \neq+\infty \\
-\infty & \text { otherwise }
\end{array}\right.
$$

It assigns the maximum grey value where it is possible to fit both SEs.

Soille's definition given in [10] is an extension to functional SE of the initial version proposed in [1].

$$
S H M T_{[F, B]}(I)(p)=\max \left\{(I \ominus F)(p)-\left(I \oplus B^{*}\right)(p), 0\right\}
$$

We can observe that Soille's and Ronse's approaches are very similar. Soille's definition returns the difference between the highest and lowest values where it is possible to fit the SEs. A unified formulation of these two approaches has been proposed in [10]. These approaches are adapted to perform pattern matching in quite good signal noise ratio environments, as we will discuss in the next section.

\subsubsection{Barat et al's $H M T$}

Barat et al introduced in [3] an operation called morphological probing that is defined by:

$$
\operatorname{BHMT}_{[F, B]}(I)(p)=\left(I \oplus B^{*}\right)(p)-(I \ominus F)(p)
$$

It measures the distance between the best inner fit of $F$ and the best outer fit of $B$. The lower is this value the best is the fit. This method presents no special noise related issue. One can note that there is a direct link between Barat's and Soille's HMT:

$$
S H M T_{[F, B]}(I)(p)=-\min \left\{B H M T_{[F, B]}(I)(p), 0\right\}
$$

thus Soille's HMT can be seen as a constrained version of Barat's HMT. 


\subsubsection{Khosravi-Schaefer's HMT}

Khosravi and Schaefer introduced in [16] another version of HMT which uses a single SE and is equivalent to Barat et al's HMT. It is defined by:

$$
\begin{aligned}
\operatorname{KHMT}_{[F]}(I)(p) & =(I \ominus F)(p)-(-I \ominus(-F))(p) \\
& =(I \ominus F)(p)-\left(I \oplus F^{*}\right)(p) \\
& =-B H M T_{[F, F]}(I)(p)
\end{aligned}
$$

KHMT is always less or equal to 0 which is reached for a perfect match.

\subsection{Generic solution to improve noise robustness}

We place this study in the context of very noisy images, considering that images are generally affected by impulsive (or speckle), Gaussian or Poisson noise, we will first review the different generic solutions that have been proposed to deal with these kinds of noise. Another aspect of noise is the inherent slight variations or uncertainties of a real signal to the ideal model. These variations can be of different types: deformations (local or global), overlapping with other objects.

A simple solution to improve the tolerance to impulsive noise of all methods is to use rank operators instead of traditional erosion and dilation [16][17]. However this solution implies to determine an appropriate rank for both dilation and erosion operations. Khosravi and Schafer [16] have shown that a lower and upper bounds for the rank are given respectively by impulsive and Gaussian noise, but they did not provide a general formula to obtain these bounds. They also concluded that the effect of rank operator on Gaussian or Poisson noise is limited. Moreover impulsive noise can be strongly reduced by applying a rank filter (e.g. a median filter) before the pattern matching operator with negligible loss of quality [18].

Bloomberg and Maragos [17] proposed several solutions to improve robustness to noise and shape uncertainties. The first idea is to perform a subsampling of both image and SEs. This solution is attractive (by considering less pixels and a Gaussian white noise, the probability of having a very noisy pixel under the SE is decreased), reduces small shape variations and saves computation time. Nevertheless it requires the resolution to be good enough so the subsampling would preserve the major features of the shape. Another possibility is to perform a spatial decimation of the support of the structural functions following a regular grid. The advantages of this method 
are the same as subsampling's ones, but it can be applied even at low resolution. Despite their capacities, these two methods are not sufficient to deal with very noisy images.

It is also possible to improve noise resistance of the morphological HMT, by first dilating both foreground and background before performing erosions, as proposed by Bloomberg [17] in the binary case. In this case the foreground and the background overlap, thus it becomes easier to fit the SEs. However the extension to the greyscale case is not trivial since we have to define the functional SE to be used.

Another way to improve robustness against noise and shape uncertainties is to provide less informative SEs (i.e. increase distance between foreground and background). This is nearly equivalent to previous Bloomberg method and can be the easiest way to work with low level SNR. Nevertheless this method fails at very low SNR as the SEs become so blurred that almost every configuration can provide a good match.

\section{Robust Hit-or-Miss}

Khosravi and Schaefer have widely tested the use of KHMT with general rank order operators face to impulsive and Gaussian noise at various SNR. They explain the difficulty to find a suitable rank which is highly dependent on noise level and type. They have also explored partitioning of the template which is a kind of variation of the decimation method. Their conclusion joined Bloomberg's one saying that it should be done following a regular grid to obtain best performances. Nevertheless with all these improvements, performances still fall when the SNR goes under $19 d B$ [16].

The intrinsic difficulty of Soille's and Ronse's HMT to deal with high noise level is due to their rigid behaviour. Because we have no intermediate level between a true answer (object is detected at point $p$ ) and a false one (object is not detected) it is really hard to find the good SEs $F$ and $B$ to be used. In fact, in these HMT the main feature that provide a robustness to noise is the distance taken between $F$ and $B$, the larger it is the more noise they can absorb and inversely. If $F$ and $B$ are too precise, too close then the transform nearly always gives a negative result. On the contrary, if $F$ and $B$ are too simple and distance between each other is too large, this will lead to a high number of false positive.

Barat et al's HMT or Khosravi-Schaefer's HMT are quite different because they evaluate a distance, thus they look more flexible. Let us consider 
a given pixel expected to return a positive result (ground truth is available). However applying Barat et al's HMT with SE $F$ and $B$ on this pixel may return a positive value $t$ instead of a negative one. Then to ensure a positive result with Ronse's or Soille's HMT at this point we have to increase the distance between the two SE's by $t$ (e.g. $F^{\prime}=F-t$ and $B^{\prime}=B$ or $F^{\prime}=F$ and $\left.B^{\prime}=B^{\prime}+t\right)$.

To sum up, Soille's and Ronse's HMT noise robustness is mainly determined by the distance between the two SEs. In Barat et al's HMT or Khosravi-Schaefer's HMT the distance between the two SEs is not important as it only results in a shift of the result. Thus the problem of determining a good distance is transformed in a problem of thresholding. The important point is to understand that these problems are equivalent and that all these formulations will react similarly when a lot of noise is present. None of them will deal with this case accurately. Figure 2 illustrates the behaviour of the various HMTs when SNR is decreasing.

In the binary case Maragos in [19] had the idea to introduce a fuzzy HMT that allows to keep well defined SEs, close to each other and that works in noisy environments. It measures a ratio between the number of pixels that actually fit in the SEs and the total number of pixels contained in the SEs. Hence, instead of looking for positions where the two SEs perfectly fit the image, we measure how well the SEs fit the image everywhere.

This can be extended to grey level images. For this we consider that the foreground and background SEs describe respectively a local lower and upper bound for our image. The fitting process measures the ratio between the number of pixels in the neighbourhood between these two bounds and the total number of pixels in the area covered by the SEs. The result is a score between 0 (no points of the neighbourhood fits in the bounds) and 1 (all pixels of the image are between lower and upper bounds).

Whereas in Ronse's or Soille's approach the distance between SEs $F$ and $B$ and so their global shape has to be modified to tolerate noise, in this approach SEs can keep a well defined shape and tolerate fluctuations due to noise.

\subsection{Definition}

We now assume that $T$ is an infinite set of grey values with a total ordering relation noted $\leq($ e.g. $T \subset \mathbb{Z})$. Formally we have to decompose the image $I$ and pair of SEs $(F, B)$ as suprema of impulses and then consider each triplet 
$(I, F, B)$ of impulses independently. Let $i_{(p, t)}$ be an impulse at point $p \in E$ of level $t \in T$ :

$$
\forall x \in E, \quad i_{(p, t)}(x)=\left\{\begin{array}{cl}
t & \text { if } p=x \\
-\infty & \text { otherwise. }
\end{array}\right.
$$

For practical reasons and because the positions of the impulses have no influence on the following definitions we write $i_{t}$ for $i_{(0, t)}$. Let us write $I C_{F, B}^{t}(q)(v)$ the proposition "pixel value $v$ is comprised between $F+t$ and $B+t$ at point $q$ in the SE", we have

$$
\begin{aligned}
I C_{F, B}^{t}(q)(v) & \Longleftrightarrow i_{F(q)+t} \leq i_{v} \leq i_{B(q)+t} \\
& \Longleftrightarrow i_{v} \oplus i_{B(q)+t} \leq t \leq i_{v} \ominus i_{F(q)+t}
\end{aligned}
$$

A proof of equivalence between Eq. 10 and 11 can be found in [10].

Let us define $S=\operatorname{supp}(F) \cup \operatorname{supp}^{*}(B)$ and card is the cardinality of a set. The proposed fuzzy HMT is then defined by:

$$
F T_{[F, B]}(I)(p)=\max _{t \in T} \frac{\operatorname{card}\left\{q \in S \mid I C_{F, B}^{t}(q)(I(p+q))\right\}}{\operatorname{card}(S)}
$$

hence it evaluates the best ratio of pixels satisfying the requirements of proposition 11 with SEs $F$ and $B$ translated at all possible grey levels.

\subsection{Implementation issues and parameters selection}

Application of this operator is not straightforward. Indeed $T$ is theoretically an infinite and perhaps continuous set, thus it is not directly possible to determine at which level $t$ the score in Eq. 12 will be maximized. In fact the interval of possible values for $t$ can be restricted quite easily by a local analysis of pixel values. For example in Fig. 3, we can estimate that the upper part of our pattern should be between grey levels 6 and 10, and this can obviously be done by applying a simple mean filter and keep the resulting value as an estimation of $t$. One can then compute score for different values near this estimation. This solution obviously depends on the possibility to extract a main feature from the pattern (for example "the peak" in Fig. 3) that can be used to perform a first and quick estimation of $t$.

Another issue is how to determine ideal distance between the two SEs. Indeed it can be estimated from local noise information. For example assuming that our image is corrupted by a Gaussian noise $\mathcal{N}\left(0, \sigma^{2}\right)$, and that we are looking for a pattern given by function $P$ : we can set $F=P-\sigma$ and $B=P+\sigma$. Then, according to noise statistic we can expect to have a matching score of about $68 \%$ in a correct case. 


\section{Application to astronomical images}

Since the discovery of the various surface brightnesses of galaxies, astronomers have become more and more interested in a way to automatically detect very faint objects in astronomical images. LSB [9] galaxies have a central surface brightness higher than $22.5 \mathrm{mag} / \operatorname{arcsec}^{2}$ (the magnitude is an inverse logarithmic brightness scale used in astronomy because of its similarities with the human eye behaviour, the arcsec is an angular distance). Traditional method of sigma clipping (i.e. adaptive thresholding method) used commonly in astronomy is not able to detect such objects as they are eliminated with the background (see Sec. 4.2 for details on sigma clipping method.)

When searching for objects with good SNR, astronomical images are quite easy to describe because they are mainly composed of bright objects on a dark background. Unfortunately several problems arise when approaching background signal level (i.e. looking at the early universe or at low brightness objects). Background is non flat over all the image, even in small images of about $512 \times 512$ pixels. Images are also affected by a Poisson noise (approximated by a Gaussian noise of mean 0 and deviation $\sigma$ ) and some salt and pepper noise. This last one may be caused by defect pixels on the CCD camera or more usually by cosmic rays. All these properties make the problem of LSB galaxies detection hard to solve (see Fig. 6 and 7.)

The whole process of LSB galaxies detection is decomposed in two steps: first a segmentation map of interesting objects is built, and then the objects present in segmentation map are characterized using physical criteria. Astronomers of the Centre de Données astronomiques de Strasbourg ${ }^{1}$ (CDS) have recently developed such a tool that performs an automatic characterisation of astronomical objects [20]. This tool has been tested to detect LSB galaxies based on segmentation map obtained using a Markovian quadtree segmentation tool [21]. The Bayesian approach is useful to involve a prior on noise but does not deal with large shape knowledge, thus we proposed a new method to compute more selective segmentation map using FHMT.

In this section we describe a method based on FHMT to automatically build a segmentation map of potential LSB galaxies. The algorithm is decomposed into several steps. First we need to build several patterns corresponding to LSB galaxies of various shapes and orientations. Because the

\footnotetext{
${ }^{1}$ http://cdsweb.u-strasbg.fr/
} 
objects we are looking for are very close to the background in terms of photometry, we also need to compute a precise map of the background (i.e. evaluate the intrinsic luminosity of the sky at all points.) Next the original image is preprocessed with a median filter to reduce noise. Filtered image, background map, and pattern set are then used to calculate FHMT. The result is thresholded and the original shape of LSB galaxies is reconstructed.

\subsection{Description of patterns}

We now describe how LSB galaxies are modelled. They are elliptic objects with a luminosity profile roughly following an exponential law. Let us consider a two dimensional discrete plane $E$. Let $\left(c_{x}, c_{y}\right) \in E$ be the centre of the galaxy, then we have for all $p \in E$ the following expression for the luminance:

$$
L S B\left(p_{x}, p_{y}\right)=\mu_{0} e^{-\frac{1}{R_{0}} \sqrt{\left(p_{x}-c_{x}\right)^{2}+b *\left(p_{y}-c_{y}\right)^{2}}}
$$

where $\mu_{0}$ is the central surface brightness given in pixel value unit, $R_{0}$ is the scale length, $b \in] 0 ; 1]$ defines the ellipticity of the model. Using this model we are able to generate several patterns with various brightnesses, elongations, orientations and scale lengths.

Because of the non constant background and the huge number of possible luminosities we choose to generate patterns at a normalized brightness $\left(\mu_{0}=\right.$ 1 ) and dynamically stretch patterns depending on local background value and noise statistics. Since there is a high correlation between the noise standard deviation $\sigma_{n}$ and magnitude scale, brightness $t$ will then be expressed as a factor $k$ of the noise deviation: $t=k * \sigma_{n}+\mu_{b}$ with $\mu_{b}$ the background level.

We considered scale length from 1.33 to 10.33 arcsec by step of 1.0 arcsec (the equivalence between arcsec and pixel unit can be computed from image file header). We quantify 14 orientations between 0 and $\pi$. The final set of SEs was obtained by combining all possible scale lengths, elongations and orientations while avoiding identical cases induced by symmetries. It is composed of 640 templates (see for example ${ }^{2}$ Fig. 4 and 5.)

All SEs are then convolved with a Gaussian kernel of $5 \times 5$ pixels to reproduce the point spread function (PSF) of the telescope (seeing and optics deformations). This is an approximation because PSF depends on the image and the location in the image, but this approximation is negligible in comparison with shape uncertainties.

\footnotetext{
${ }^{2}$ All images are given in inverse grey level to save ink.
} 
Finally each pattern is composed of two SEs of same orientation and elongation. We choose to take the foreground SE to have a scale length smaller of 1 arcsec than the background one. The distance between the SEs is adjusted dynamically with respect to local noise statistics (see Sec. 4.2.).

\subsection{Computation of FHMT}

Once we have generated all different patterns, we estimate background and noise of the image by following a regular grid of $32 \times 32$ pixels in a window of $256 \times 256$ pixels with the sigma clipping method widely used in astronomical imaging. This method performs in two steps: in the first one it computes the average value $\mu$ (mean or median) and deviation $\sigma$ of pixels in the estimation window, then in the second step, it masks all pixels for which value is greater (resp. lower) than $\mu+k \sigma$ (resp. $\mu-k \sigma$ ), and goes back to step one until convergence. Parameter $k$ gives the confidence interval with respect to noise power, a value of 3 is generally used ${ }^{3}$. Background and noise estimation cannot be performed over all pixels because of its prohibitive computation time. Nevertheless as these parameters vary slowly over the image, we can have a good estimation of them by simply interpolating values of the background grid.

Computation of scores is done according to principles given in Sec. 3.2. Estimation of the best grey level $t$ for the vertical translation of the SEs is done using a median filter $(5 \times 5)$. We transpose foreground (resp. background) SE to local background level and stretch it so that its highest value is $t-\sigma$ (resp. $t+\sigma$ ) with $\sigma$ the local deviation of noise. Matching scores between each pattern and median filtered image is then computed and the best one is kept as the result (Fig. 6 and 7).

\subsection{Reconstruction of score map}

In the next step, the score map is thresholded. The threshold value offers a good tuning option and was determined to minimize false negative rate and, with less importance, minimize false positive rate. We found that a score of $80 \%$ offers a good compromise. The use of the same threshold for every observation is not a problem. As all parameters of the algorithm are set automatically according to observation parameters and statistics, the score gives an absolute measure which is independent of the observation.

\footnotetext{
${ }^{3}$ See Sextractor documentation for details: http://terapix.iap.fr/
} 
Once we have a binary map, we need to reconstruct the final map. To do this, each pixel of the binary map is dilated by the support of the pattern that gives the maximum score in this position (Fig. 6 and 7). Scheme of the procedure is given in Fig. 8.

To improve map's precision we could have used a geodesic reconstruction instead of a simple dilation so the reconstructed areas would exactly fit detected objects. In our case this is useless because the next step of the algorithm rather prefers to work with idealised shapes instead of precise but noisy boundaries.

\subsection{Optimisation}

Without any optimisation the proposed algorithm takes about 20 minutes to compute the segmentation map on an image of $512 \times 512$ pixels. This time includes estimation of background and generation of the 640 patterns. The code is written in Java and executed on a Pentium IV computer. In this section we propose several optimisations to improve algorithm performances:

- We perform a spatial decimation of patterns using Bloomberg's method [17], we choose to keep one pixel every two;

- As the resolution is sufficient in our images, we perform segmentation in a sub-grid of the images at scale 1:2;

- Score computation and thresholding are done simultaneously, so the computation of a fit can be discarded as soon as threshold becomes unreachable;

- Last optimisation is heuristic and comes from the idea that we can perform the segmentation in two steps. The first step will make a fast selection of possibly interesting pixels, second step will perform a full computation for selected pixels. During the first step we will segment the images using only a few patterns (we take only circular patterns, orientation and elongation are not considered) and a low threshold (60\%). During second step we segment pixels selected at first step with the full process (all patterns, threshold at 80\%).

With all these optimisations we are able to compute a segmentation map of a $512 \times 512$ images in about 2 minutes, that is ten times faster than original algorithm. Detection maps obtained with and without optimisation 
are nearly the same. One can notice that last optimization is purely heuristic and so we cannot assert that target will never be lost. However we have compared results obtained with both the original strategy and this 2-step approach, and we have observed only minor differences (small difference in the shape or size of the detected targets). In these experiments no target were lost.

\section{Results}

In this section, the method was evaluated through two different datasets. First, to compensate the lack of ground truth in real images, we created a simulated dataset. However we also involved a set of real images which have already been analysed by experts [22] to show the relevance of the proposed approach in this context. Due to the lack of related work and the clear inability of existing HMT to deal with this problem (as shown in Sec. 3), evaluation can rely only on quantitative and qualitative analysis (and thus cannot involve any comparison).

\subsection{Simulated dataset}

It is very important for astronomers to know the detection limits of the algorithm they are using. This information is used to determine the statical bias of their conclusions due to selection effects of the algorithm.

A batch of tests has been designed with astronomers of the CDS to determine the detection limits of our algorithm in terms of brightness and scale length. These tests apply on simulated images of LSB galaxies for which we know the ground truth. The algorithm used to generate artificial LSB galaxies is nearly the same as the one used to build the SEs in the detection algorithm. We used a Gaussian function to reproduce PSF. We also had the possibility to add other astronomical objects in our images (like stars or bright galaxies) to test robustness of our algorithm in crowded part of the sky. Noise power and pixels statistics used to create images were taken directly from a real astronomical image. Figure 9 shows examples of simulated images of LSB galaxies.

Our test protocol is divided into three parts (Fig. 9), we applied the algorithm on:

- images containing only one LSB galaxy (one test per couple (brightness, radius)) to determine absolute limits; 
- images containing one LSB galaxy and a bright star (one test per couple (brightness, radius)) to study reaction in a sparse environment;

- images containing one LSB galaxy and between 3 and 7 other objects located randomly over the images (21 tests per couple (brightness, radius)) to test robustness in crowded environment.

LSB galaxies do not have strict boundaries, thus we choose to define their limits as the ellipse where the signal has lost $95 \%$ of its central energy. The area enclosed in this radius gives us the reference truth to evaluate the map proposed by the detection algorithm. Let $C_{r e f}$ be the set of pixels present in the reference map. There may be several objects in the segmentation map provided by the method, thus we choose to keep the connected component having the largest intersection with the reference map as the final result and denotes it by $C$.

Finally we used a F1-measure [23] to evaluate correctness of the detection. The later combines the precision and the recall measures to obtain a score between 0 and 1 . It is given by:

$$
\mathrm{F} 1-\text { measure }=\frac{2 * \text { precision } * \text { recall }}{\text { precision }+ \text { recall }}
$$

with precision defined as:

$$
\text { precision }=\frac{\operatorname{card}\left(C \cap C_{r e f}\right)}{\operatorname{card}(C)}
$$

and recall defined as:

$$
\text { recall }=\frac{\operatorname{card}\left(C \cap C_{r e f}\right)}{\operatorname{card}\left(C_{r e f}\right)}
$$

Experiments have shown that a F1-measure value equal to at least 0.4 is sufficient for the following steps to perform accurately.

We have determined that the FHMT algorithm is able to detect LSB galaxy up to a scale length of 2 arcsec (this is PSF limit anyway) and a central surface brightness of $\sim 26.2 \mathrm{mag} / \operatorname{arcsec}^{2}(0.7 \sigma$ over sky background level which is nearly equivalent to $0 d B$ SNR). Moreover detection is nearly not affected by other objects except if the wing of another bright object covers the LSB galaxy (the two objects overlap and the LSB galaxy is drown in the other object luminosity). 
We have also compared the results of our method to those obtained with a traditional correlation method [18]. This was done by replacing the FHMT measure by the correlation one and adjusting the threshold value in the process to ensure the best possible results. The correlation based method performs well until $1.5 \sigma$ over sky background level (Fig. 10), i.e. its robustness to noise is lower than the FHMT one. This shows that (robust) morphological approaches can be relevant alternatives to statistical methods even in such noisy environments.

\subsection{Real dataset}

Next the FHMT operator was applied on a test set of 16 blue band (a filter centred at wavelength of 4500 Angström corresponding to blue colour is used) images of $2048 \times 4096$ pixels coming from the INT Wide Angle Survey ${ }^{4}$. The dataset covers the Virgo cluster which has the advantage to be close to the Galaxy and in a relatively sparse area of the sky. Moreover the population of LSB galaxies in this survey has already been studied by astronomers [22], which gives us a reference catalogue to evaluate our results.

The algorithm has been run over the whole dataset and segmentation maps were used as inputs for the characterisation tool of the CDS: DetectLSB. At the end of the process, this tool provides HTML files describing potential LSB galaxies found and XML catalogues in the VOTable standard $[20]$.

A full analysis of the result for two images was performed in collaboration with an astronomer. The reference catalogue contains 9 LSB galaxies in these areas. The algorithm proposed 23 candidates. It founds 6 objects of the reference catalogue and provided 8 new LSB galaxies. The spurious detections were mainly due to artefacts created by two close sources (when wings of two close objects intersect, they locally create an artificial raise of luminosity) or very distant galaxies (the brightness of the galaxy is then decreased by absorption of intergalactic environment). That leads to a total of 17 interesting objects (9 already known and 8 newly discovered) for the 2 images. The recall of the method is then 0.82 and the precision is 0.6. One can note that the 3 LSB galaxies missed from the reference catalogue were correctly segmented by FHMT but were rejected by the characterisation algorithm. Because LSB galaxies detection is a very challenging task for

\footnotetext{
${ }^{4}$ http://www.ast.cam.ac.uk/ rgm/int_sur/
} 
which no automatic solution has been proposed yet, these scores can be considered as really good.

The traditional correlation method performed on these two images, using the same methodology as in section 5.1, proposed 54 candidates. It founds 9 of the 17 LSB galaxies previously mentioned, yielding a recovery rate equals to $53 \%$. The false positive were mainly multiple detections (two or more close objects are fused in a single one in the final map) or very distant galaxies.

A comparison of the new catalogue for the whole set of 16 images with the existing one gives us a recovery rate of $87 \%$ and several new candidates. These candidates have now to be analysed by astronomers' community before any conclusion can be drawn on them.

\section{Conclusion}

We have addressed the particular problem of template matching in very noisy environment using the morphological HMT. We have shown that the existing formulations of HMT are not adapted to do this, thus we proposed a formulation of a "fuzzy" HMT. It relies on two SEs: the foreground and the background one and consists of measuring the ratio of the image area that fit the SEs compared to the total area covered by the SEs.

We have described how it can be applied to a real and still unsolved case in astronomical imaging: the automatic detection of LSB galaxies. Our results have been analysed by an astronomer and confirmed that FHMT is suitable for pattern matching at very low SNR.

Thereby the field of application of the morphological HMT has been extended to very noisy images for which statistical approaches are generally preferred. The process we described can be easily adapted for pattern matching in other domains such as radar imaging. Moreover, in case of noisy multispectral data, the proposed operator should be extended to multivariate mathematical morphology [24].

\section{Acknowledgment}

The authors thank Bernd Vollmer (Observatoire de Strasbourg, Universit de Strasbourg), François Bonnarel (Centre de Donnes astronomiques de Strasbourg, Universit de Strasbourg), Mireille Louys (Laboratoire des Sciences de l'Images, de l'Informatique et de la Tldtection, Universit de Strasbourg) and Wim Van Driel (Observatoire de Meudon, Institut d'Astrophysique 
de Paris) for their enlightening discussion about LSB galaxies and the various aspects of astronomical image analysis.

\section{References}

[1] P. Soille, Morphological Image Analysis: Principles and Applications, Springer-Verlag New York, Inc., Secaucus, NJ, USA, 2003.

[2] B. Naegel, N. Passat, C. Ronse, Grey-level hit-or-miss transforms-part ii: Application to angiographic image processing, Pattern Recognition 40 (2) (2007) 648-658.

[3] C. Barat, C. Ducottet, M. Jourlin, Pattern matching using morphological probing., in: International Conference on Image Processing (1), 2003, pp. 369-372.

[4] S. Lefèvre, J. Weber, D. Sheeren, Automatic building extraction in VHR images using advanced morphological operators, in: IEEE/ISPRS Joint Workshop on Remote Sensing and Data Fusion over Urban Areas (URBAN), Paris, 2007.

[5] A. Puissant, J. Weber, S. Lefèvre, Coastline extraction in VHR imagery using mathematical morphology with spatial and spectral knowledge, in: ISPRS International Congress, Beijing, 2008.

[6] D. S. Bloomberg, L. M. Vincent, Pattern matching using the blur hitmiss transform, Journal of Electronic Imaging 9 (2000) 140-150.

[7] B. Raducanu, M. Graña, A grayscale hit-or-miss transform based on level sets, in: International Conference on Image Processing, 2000, pp. 931-933.

[8] E. Aptoula, S. Lefèvre, C. Collet, Mathematical morphology applied to the segmentation and classification of galaxies in multispectral images, in: European Signal Processing Conference (EUSIPCO), 2006.

[9] G. Bothun, C. Impey, S. McGaugh, Low-Surface-Brightness Galaxies: Hidden Galaxies Revealed, Publication of the Astronomical Society of the Pacific 109 (1997) 745-758. 
[10] B. Naegel, N. Passat, C. Ronse, Grey-level hit-or-miss transforms-part i: Unified theory, Pattern Recognition 40 (2) (2007) 635-647.

[11] H. J. A. M. Heijmans, Theoretical aspects of gray-level morphology, IEEE Transaction on Pattern Analysis and Machine Intelligence 13 (6) (1991) 568-582.

[12] C. Ronse, Why mathematical morphology needs complete lattices, Signal Processing 21 (2) (1990) 129-154.

[13] J. Serra, Morphological image operators, Society for Industrial and Applied Mathematics Review 38 (1) (1996) 178-179.

[14] S. R. Sternberg, Grayscale morphology, Computer Vision, Graphics, and Image Processing 35 (3) (1986) 333-355.

[15] C. Ronse, A lattice-theoretical morphological view on template extraction in images, Journal of Visual Communication \& Image Representation 7 (3) (1996) 273-295.

[16] M. Khosravi, R. Schafer, Template matching based on a grayscale hitor-miss transform, IEEE Transactions on Image Processing 5 (5) (1996) 1060-1066.

[17] D. S. Bloomberg, P. Maragos, Generalized hit-miss operations, in: SPIE Conference 1350, Image Algebra and Morphological Image Processing, San Diego, CA, 1990, pp. 116-128.

[18] R. Gonzalez, R. Woods, Digital image processing, ISBN: 0-20-118075-8, Prentice Hall, Englewood, 2002.

[19] P. Maragos, Optimal morphological approaches to image matching and object detection, in: International Conference on Computer Vision, 1988, pp. 695-699.

[20] M. Louys, B. Perret, B. Vollmer, F. Bonnarel, S. Lefèvre, C. Collet, Lsb galaxies detection using markovian segmentation on astronomical images, in: ASP Conference Serie, Astronomical Data Analysis Software and Systems XVII, London, UK, 2007, pp. 116-128. 
[21] C. Collet, F. Flitti, Variations on markovian quadtree model for multiband astronomical image analysis, in: International Symposium on Applied Stochastic Models and Data Analysis, Brest, FR, 2005.

[22] S. Sabatini, J. Davies, R. Scaramella, R. Smith, M. Baes, S. M. Linder, S. Roberts, V. Testa, The dwarf LSB galaxy population of the Virgo cluster - I. The faint-end slope of the luminosity function, Monthly Notices of the Royal Astronomical Society 341 (2003) 981-992.

[23] T. Y. Chen, F.-C. Kuo, R. Merkel, On the statistical properties of the f-measure, Quality Software, QSIC 2004. Proceedings. Fourth International Conference on (2004) 146-153.

[24] E. Aptoula, S. Lefèvre, A comparative study on multivariate mathematical morphology, Pattern Recognition 40 (11) (2007) 2914-2929. 


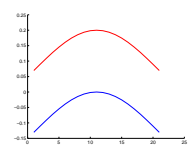

(a)

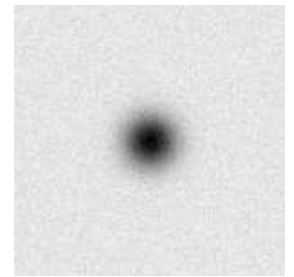

(b)

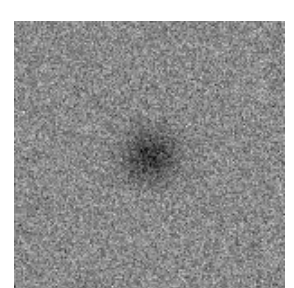

(d)

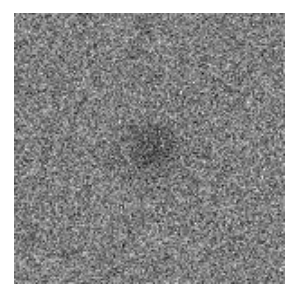

(f)

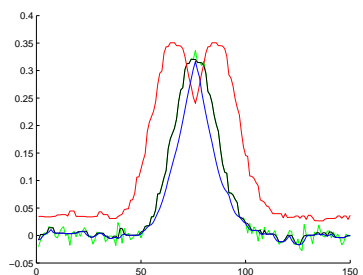

(c)

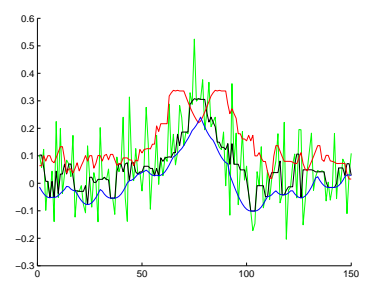

(e)

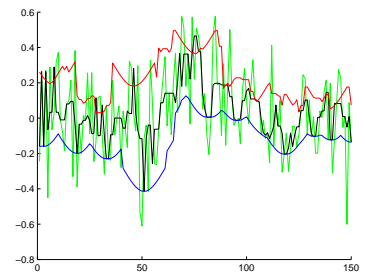

(g)

Figure 2: This figure shows how the grey dilation and erosion and consequently the various formulations of HMT react when SNR falls. In this example a round shape with a Gaussian luminosity profile is hidden in noise. The image (a) is a one dimensional cut of the foreground SE $F$ (blue curve) and background SE $B$ (red curve). Then the left column shows images with decreasing SNR: $\sim 20 d B$ for image (b), $\sim 0 d B$ for image (d) and $\sim-8 d B$ for image (f). The right column represent a one dimensional cut of the left images (in green), a cut of a median filtered (size $5^{*} 5$ ) version of the left image (black), the erosion of the filtered image by the foreground SE: $I \ominus F$ (in blue), and the dilation of filtered image by the dual of background $\mathrm{SE} I \oplus B^{*}$ (in red). In plot (c) and (e), the red curve goes under the blue one, which would give a positive result for RHMT and SHMT. In the third case the detection fails as dilation and erosion are disturbed by noise. One can note that if we increase the distance between SE $F$ and $B$ for example by defining $F^{\prime}=F-k$ with $k$ a grey value, the result would be equivalent to a vertical translation of the blue curve. And this is obviously useless as no vertical translation of the blue curve can make it cross the red curve only at the centre (i.e. if the blue line crosses the red one at another point this means that a false positive is detected.) 

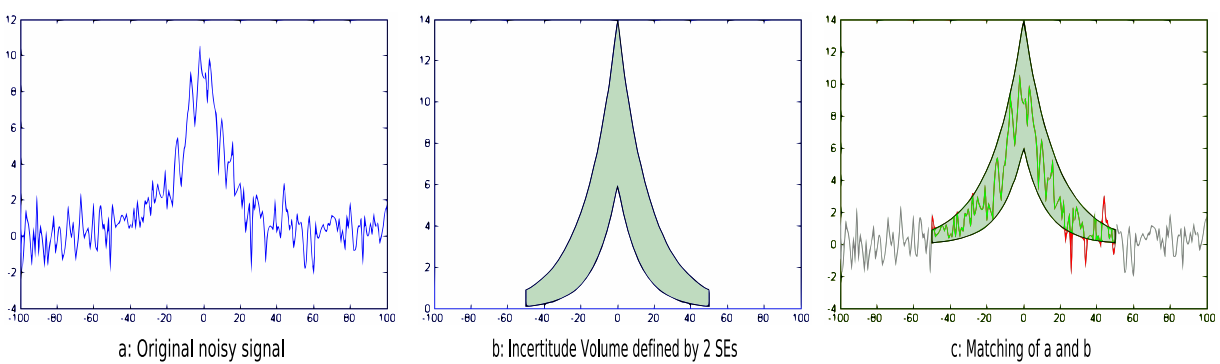

Figure 3: Example of application of the fuzzy HMT to detect an exponential profile with Gaussian noise. Image (a) represents a $1 \mathrm{D}$ noisy signal $(\mathrm{SNR} \approx 9 d B)$. Image (b) represents the uncertainty area defined by the 2 SEs $F$ and $B$. Image (c) shows how well the pattern can fit the signal.

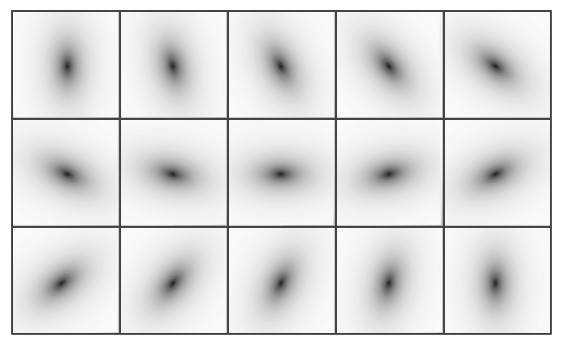

Figure 4: Example of SEs obtained with a variation of orientations convolved with a Gaussian point spread function.

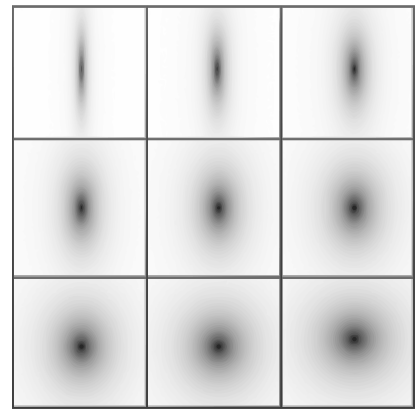

Figure 5: Example of SEs obtained with a variation of elongations convolved with a Gaussian point spread function. 


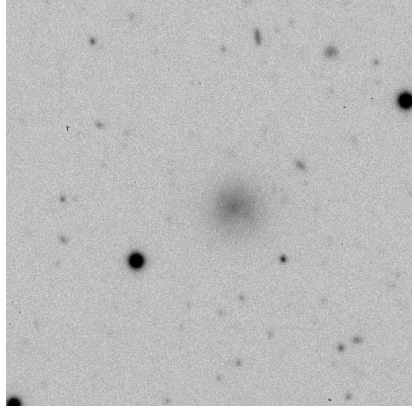

(a)

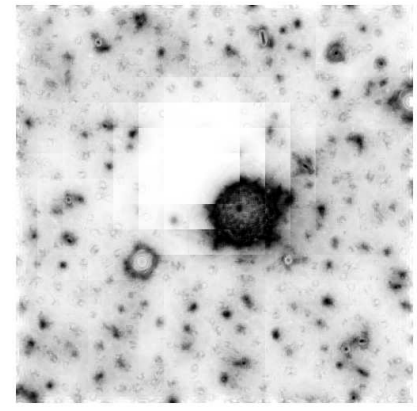

(b)

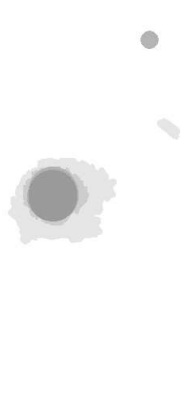

(c)

Figure 6: Application of FHMT to LSB detection, an easy case. (a) original image of $512 \times 512$ pixels with a LSB galaxy at centre. One can note that the pixel dynamic has been adjusted manually so the LSB galaxy becomes visible. (b) score map obtained after application of FHMT. The LSB is clearly visible. We can observe that both centre and wings of the object give a good score. (c) Thresholded and reconstructed map. The final map contains different classes proportional to the object luminosity. This allows deblending capabilities for overlapping objects having different luminosities.

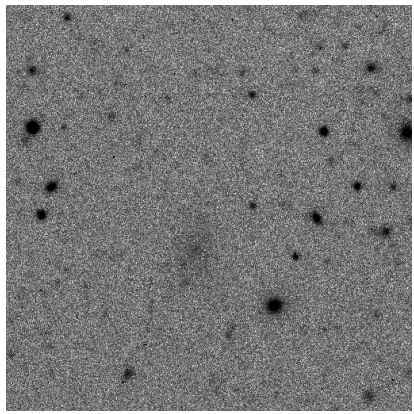

(a)

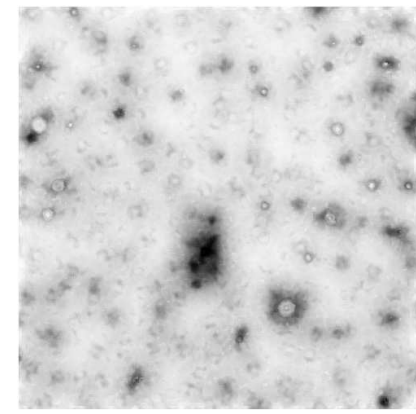

(b)

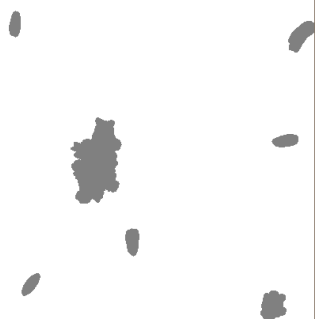

(c)

Figure 7: Application of FHMT to LSB detection, a hard case. (a) original image of $512 \times 512$ pixels with a LSB galaxy at centre. One can note that the pixel dynamic has been adjusted manually: the LSB galaxy is still hard to view (b) score map obtained after application of FHMT. The LSB is clearly visible. We can observe that the object appears to be composed of several small ellipses, that is due to the irregular aspect of the galaxy. (c) Thresholded and reconstructed map. There is clearly one false positive on this map on the top right border (it corresponds to the wings of a brighter object). Other objects detected have to be deeper analysed to determine their physical nature. 


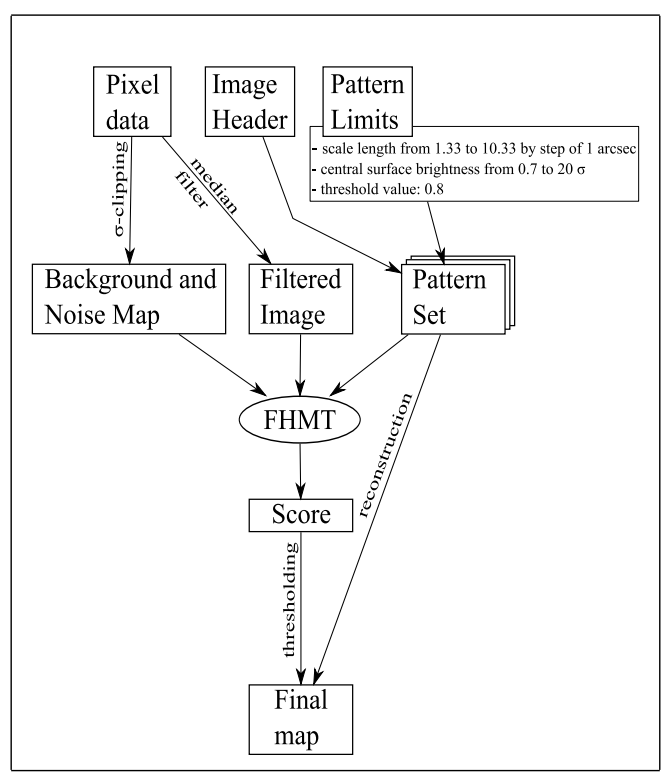

Figure 8: Scheme of the pattern matching method. The algorithm takes three inputs: image pixel values, image file header, and physical limits. The whole procedure is automatic. 

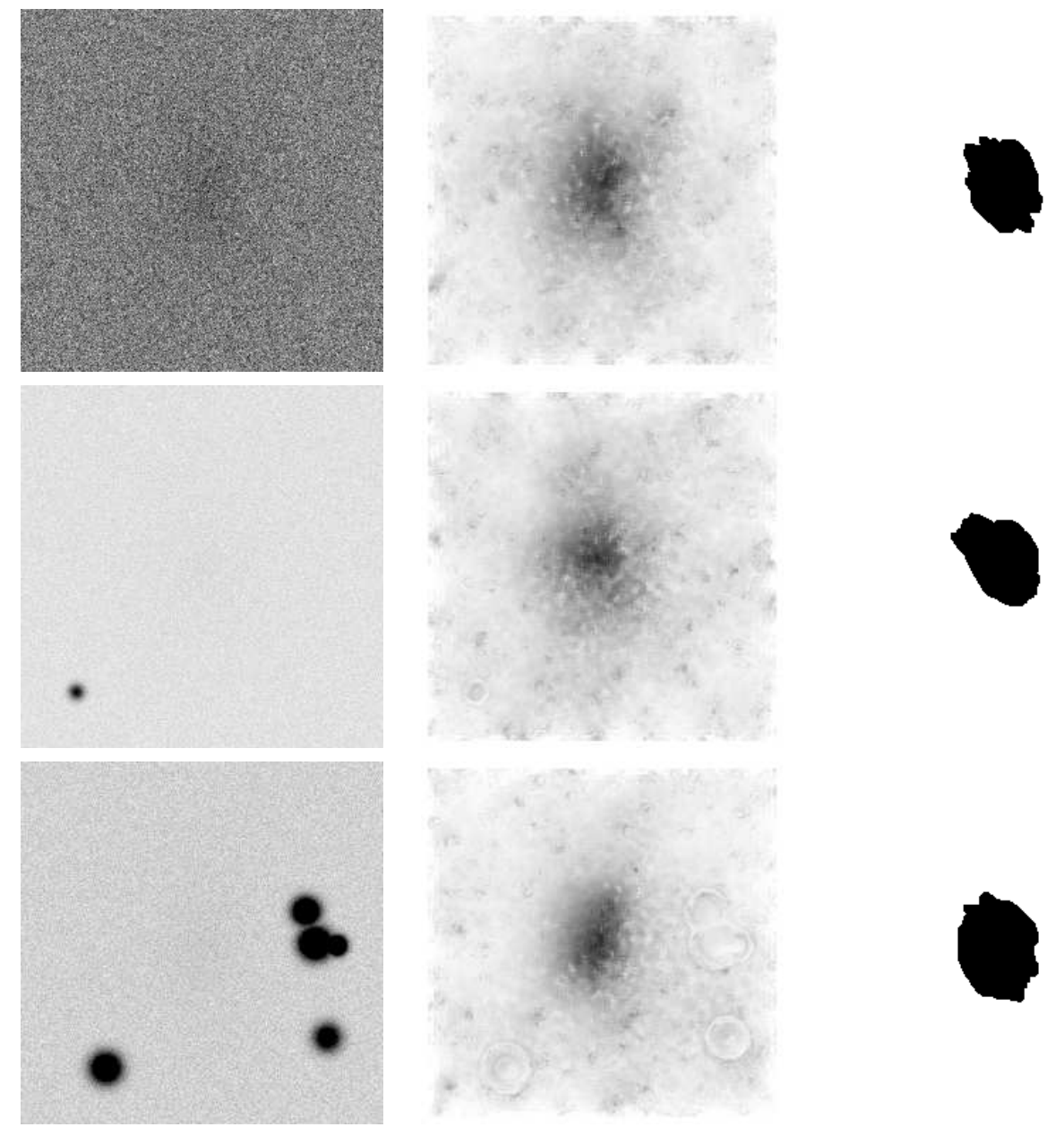

Figure 9: Example of simulated images for the three steps of our evaluation protocol. LSB galaxies displayed in the centre of each image have a scale length of 10 pixels and a central surface brightness of $25.7 \mathrm{mag} / \operatorname{arcsec}^{2}$ (one $\sigma$ over sky background level with $\sigma$ the deviation of noise). SNR is very low about $-1 d B$. First line shows results for a single LSB galaxy, second line for a LSB galaxy with a star near it, and third line for a LSB galaxy surrounded by 5 stars randomly placed. The first column shows the simulated image, the second one shows result of FHMT, and the third one the final map. 

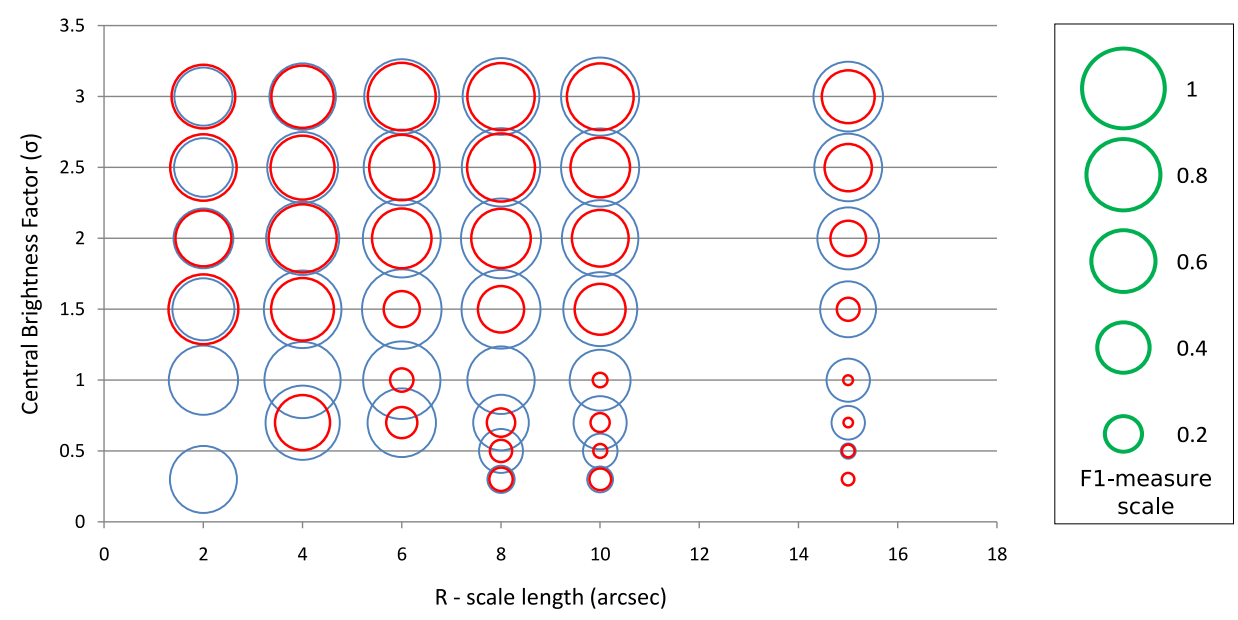

Figure 10: Comparison of the results obtained using the FHMT (blue circles) and the correlation (red circles) on simulated LSB galaxies. For each couple (radius, brightness factor), the circle area is proportional to the F1-measure value. The green circles on the right side give a reference scale. The experiments showed that the detection can be considered to be effective when the F1-measure is above or equal to 0.4. While the performances of the correlation drops under $1.5 \sigma$, the FHMT remains effective until $0.5 \sigma$ over sky background level. 